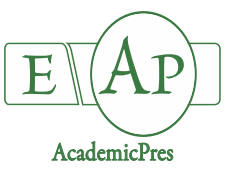

Akçura S et al. (2021)

Notulae Botanicae Horti Agrobotanici Cluj-Napoca

Volume 49, Issue 2, Article number 12224

DOI: $10.15835 /$ nbha49212224

Research Article

\title{
Effects of irrigation intervals and irrigation levels on oil content and fatty acid composition of peanut cultivars
}

\author{
Sevim AKÇURA ${ }^{1}$, İsmail TAŞ², Kağan KÖKTEN³, \\ Mahmut KAPLAN ${ }^{4 *}$, Aydın Ş. BENGÜ ${ }^{5}$ \\ ${ }^{\text {I} C ̧ a n a k k a l e ~ O n s e k i z ~ M a r t ~ U n i v e r s i t y, ~ I n s t i t u t e ~ o f ~ N a t u r a l ~ a n d ~ A p p l i e d ~ S c i e n c e s, ~ F i e l d ~ C r o p s ~ D e p a r t m e n t, ~ C ̧ a n a k k a l e, ~ T u r k e y ; ~}$ \\ sevimakcura@yahoo.com \\ ${ }^{2}$ Çanakkale Onsekiz Mart University, Agricultural Faculty, Agricultural Structures and Irrigation Department, Çanakkale, Turkey; \\ tas@comu.edu.tr \\ ${ }^{3}$ Bingöl University, Agricultural Faculty, Field Crops Department, Bingöl, Turkey; kkokten@bingol.edu.tr \\ ${ }^{4}$ Erciyes University, Agricultural Faculty, Field Crops Department, Kayseri, Turkey; \\ mahmutk@erciyes.edu.tr (*corresponding author) \\ ${ }^{5}$ Bingöl University, Vocational College of Health Services, Medical Services and Techniques Department, Bingöl, Turkey; \\ abengu@bingol.edu.tr
}

\begin{abstract}
Oil content and fatty acid composition are the most significant quality criteria of peanuts (Arachis hypogaea L.), and these parameters is greatly influenced by irrigation and fertilization practices. A study was conducted to investigate the effects of irrigation intervals and irrigation levels on oil content and fatty acid composition of peanuts, under sandy soil conditions in two consecutive years, using 'Halisbey', 'NC-7', and 'Sultan' peanut cultivars, commonly grown in Turkey. Irrigation levels were arranged based on total evaporation from Class-A pan, and irrigations were applied through drip lines. Irrigation intervals were set as two and four days, and irrigation levels were set as $50 \%\left(\mathrm{I}_{50}\right), 75 \%\left(\mathrm{I}_{75}\right), 100 \%\left(\mathrm{I}_{100}\right)$ and $125 \%\left(\mathrm{I}_{125}\right)$ of Class-A pan evaporations. Oil content, unsaturated fatty acids (oleic and linoleic acids), and saturated fatty acids (palmitic, myristic, arachidic, behenic and lignoceric acids) were determined. For oil content, treatments were identified as the most appropriate irrigation for a two-day irrigation interval of all cultivars, $\mathrm{I}_{100}$ for four-day irrigation interval of 'Halisbey' and 'Sultan' cultivars and $\mathrm{I}_{75}$ for four-day irrigation interval of 'NC-7' cultivar. Oleic, linoleic, and palmitic acids were the major fatty acids of peanuts. Cultivars exhibited different variations in these fatty acids based on irrigation intervals and irrigation levels. In general, oleic acid contents decreased, but linoleic and palmitic acid contents increased with increasing irrigation levels. The greatest oleic acid contents were obtained from two and four-day irrigation intervals of $\mathrm{I}_{50}$ treatments in 'Halisbey' and 'NC-7' cultivars and from two and four-day irrigation intervals of $\mathrm{I}_{75}$ treatments of the second year in 'Sultan' cultivar. Present findings revealed that for quality peanut production, both irrigation intervals and irrigation levels should be taken into consideration.
\end{abstract}

Keywords: Arachis hypogaea L.; fatty acid composition; irrigation interval; irrigation level; oil content; peanut

Received: 15 Jan 2021. Received in revised form: 25 Mar 2021. Accepted: 16 Apr 2021. Published online: 29 Apr 2021.

From Volume 49, Issue 1, 2021, Notulae Botanicae Horti Agrobotanici Cluj-Napoca journal uses article numbers in place of the traditional method of continuous pagination through the volume. The journal will continue to appear quarterly, as before, with four annual numbers. 


\section{Introduction}

Irrigation is an essential component of plant production. Proper irrigation programs play a key role not only in improving both the yield and the quality. Irrigation programs play a significant role also in the preservation and efficient use of water resources (Kaplan et al., 2019). Increasing water demands are not sufficiently met with deficit water resources, and existing droughts generate crisis not only in agricultural productions but also in several other sectors. These cases will probably get worse in the future. Therefore, deficit water resources should be properly and efficiently be used, especially in agriculture and the other sectors. The proper and efficient application of irrigation water at the right time and the right quantity to root zone of the plants without generating any environmental problems will improve yield and quality. Plants exhibit quite different responses against water deficits (Tas, 2017). Therefore, the plant responds to water deficits that should be determined for sustainable agricultural production. Plant responses should especially be determined in peanut-like plants mostly used as industrial raw material.

Peanut is an annual leguminous plant either used for oil production in the industry or consumed as an appetizer. Peanut is an important oil crop cultivated worldwide in different continents, and world annual production is about 43.9 million tons (Sezen et al., 2019). Turkey, with available climate conditions, is among the leading peanut producer countries of Europe (Florkowski, 2012). Peanut is cultivated as the main and second crop in some regions of Turkey (Çukurova and Southeast Anatolia region) but cultivated as only the main crop in Aegean, Mediterranean, and Marmora regions. Peanut is a legume crop able to fixate $150 \mathrm{~kg} / \mathrm{ha}$ nitrogen into soils through symbiotic rhizobium bacteria in a single growing season (Arioglu, 2014). With such an attribute, the peanut is a significant crop roration and green fertilizer plant (Langat et al., 2006). Harvest residues could also be used as roughage, thus generate a significant feed source for animals (Kokten et al., 2014).

Peanut seeds are rich in proteins, essential fatty acids, minerals, and bioactive compounds (Akram et al., 2018). Oil is the major component of peanut seeds (36-54\%), and seeds also contain high quantities of proteins (16 - 36\%) and carbohydrates (10 - 20\%) (Davis et al., 2016). Peanut seeds also contain coenzyme Q10, arginine, and polysterols (Akhtar et al., 2014). However, the majority of world peanuts are produced for cooking oil just because of light and soft taste and some advantages of peanut oil over the other vegetable oils (Toomer, 2018). Following the oil extraction, the remaining peanut meal is quite rich in protein (50\%), thus constitutes an important source of nutrients (Zhao et al., 2012). Since peanut is an oil crop, characteristics of peanut oil should be known (Abbas et al., 2018). Peanut oil contains major saturated fatty acids of palmitic (C16: 0), unsaturated fatty acids of oleic (C18: 1) and linoleic acids (C18: 2) (Toomer, 2018). Besides these fatty acids, peanut oil also contains slight quantities of stearic acid (C18:0), arachidonic (C20: 0), eicosenoic (C29: 1), behenic (C22: 0) and lignoceric (C24: 0) acids (Casini et al., 2003; Carrin and Carelli, 2010).

The taste and quality of peanuts and peanut products largely depend on the chemical composition of peanut oil. With a high oleic acid content, peanut oil has a well oxidative and frying stability. Peanut oil does not provide solidity when exposed to air, but become solid between $0-3{ }^{\circ} \mathrm{C}$ (O'Brien, 2004). Lipid and fatty acid composition of peanut oil-primarily depend on the cultivar, seed maturity, environmental conditions, agronomic practices, and geographical location (Young, 1996). Low temperatures at the seed ripening stage influence the unsaturated fatty acid composition of peanuts (Casini et al., 2003). Oleic acid content increases, and linoleic and palmitic acid contents decrease toward the harvest maturity (Hinds, 1995). Andersen and Gorbet (2002) indicated that the fatty acid profile of peanuts did not effect varied with the sowing times and the years. It was indicated in another study that the fatty acid profile of peanut may vary with the soil texture (Hinds, 1995). However, it was indicated in studies conducted in the USA that genetics significantly influenced the fatty acid composition of peanuts, and greater genetic-dependent variations were observed in major components (palmitic, oleic, and linoleic acid) than the other minor fatty acids (Zhu et al., 2004).

'NC-7', 'Halisbey', and 'Sultan' are the most commonly cultivated peanut cultivars in Turkey, and they belong to the Virginia group (Arığlu et al., 2016). Oil contents of peanut cultivars grown in different environments of Turkey were reported as between 35.3-45.4\% (Özcan, 2010). In a previous experiment 
conducted in a single location, the oil content was reported as $48.94 \%$ for 'Halisbey' cultivar, $47.55 \%$ for 'NC7' cultivar, and 48.62\% for 'Sultan' cultivar (Arığlu et al., 2016). Yol et al. (2017) reported the oil content of peanut genetic materials as between 31.7 - 57.0\%, oleic acid contents as between $35.3-60.9 \%$ and linoleic acid contents as between $16.1-43.6 \%$. Greater adaptation to certain environmental conditions may contribute to high seed yield and quality of peanuts (Yol and Uzun, 2018). Growing techniques greatly influence oil content and fatty acid composition. Isleib et al. (2008) indicated that the chemical composition of peanut seeds significantly influenced by environmental factors, genetic factors, and interactions of these factors. Researchers also investigated the effects of drought, water deficit and air temperature on fatty acid composition of peanuts at different locations (Young et al., 1974; Dwivedi et al., 1996; Yao et al., 2003; Reddy et al., 2003; Patel et al., 2008; Shinde and Laware, 2010). A limited number of researchers investigated the effects of irrigation water levels on quality parameters of peanuts (Zhu et al., 2004; Amir et al., 2005; Boydak et al., 2010; Aydinsakir et al., 2018). However, a comprehensive study investigating the effects of cultivars, irrigation intervals, and irrigation levels on oil content and fatty acid composition of peanuts hasn't been conducted yet.

This study was conducted to assess the variations in oil contents and fatty acid composition of 'Halisbey', 'NC-7', and 'Sultan' peanut cultivars grown under sandy soil conditions, two different irrigation intervals, and different irrigation levels for two growing seasons. Effects of irrigation interval $\mathrm{x}$ irrigation level interactions on oil content and fatty acid compositions were also investigated, and proper irrigation practices for quality peanut production under sandy soil conditions were tried to be identified.

\section{Materials and Methods}

\section{Study area, the conditions in which the research was carried out and analyzed material}

This study was conducted for two years between May and October of 2017 and 2018 under Balıkesir Burhaniye conditions over the experimental fields of Farmer's Training and Extension Center of Balıkesir Greater City Municipality, Turkey. Irrigation-related soil parameters of the experimental site are provided in Table 1. Soil texture is mostly sandy, and sandy-loam and sand contents varied between $58.7-95.0 \%$, silt contents varied between 2.4-30.1\%, and clay contents varied between 2.6-21.4\%. Field capacity (FC) values varied between $10.87-17.78 \%$, and permanent wilting point (PWP) values varied between $4.83-8.67 \%$. Soil bulk density $(\mathrm{BD})$ values varied between $1.36-1.59 \mathrm{~g} / \mathrm{cm}^{3}$.

'Halisbey', 'NC-7', and 'Sultan' cultivars with Virginia-type seeds and commonly grown in Turkey were used as the plant material of the study. Two different irrigation intervals ( 2 and 4 days) and four irrigation levels $\left(\mathrm{I}_{50}, \mathrm{I}_{75}, \mathrm{I}_{100}\right.$, and $\left.\mathrm{I}_{125}\right)$ were applied. The amount of irrigation water was calculated based upon cumulative evaporation from Class-A-Pan (CAP). There different irrigation intervals ( 2 and 4 days) and level of cumulative evaporation $\left(\mathrm{I}_{50}, \mathrm{I}_{75}, \mathrm{I}_{100}\right.$ and $\left.\mathrm{I}_{125}\right)$ were used in the experiment. Experiments were conducted in splitsplit plots experimental design with three replications. The main plots included cultivars, sub-plots included irrigation intervals, and sub-sub-plots included irrigation levels. A $4 \mathrm{~m}$ spacing was provided between sub-plots, and $2 \mathrm{~m}$ spacing was provided between sub-sub-plots to prevent transitions between the treatments. Plots were $5 \mathrm{~m}$ long and composed of 4 rows. Row spacing was $70 \mathrm{~cm}$, and on-row plant spacing was $20 \mathrm{~cm}$.

Sowing was performed on 20 April 2017 in the first year and 23 April 2018 in the second year. Two seeds were sown manually into each seedbed, and following the emergence, the number of plants was thinned to one in each seedbed. Before sowing, $250 \mathrm{~kg} / \mathrm{ha} \mathrm{DAP}\left(45 \mathrm{~kg} / \mathrm{ha} \mathrm{N}, 115 \mathrm{~kg} / \mathrm{ha} \mathrm{P}_{2} \mathrm{O}_{5}\right)$ fertilizer was provided, and $50 \mathrm{~kg} / \mathrm{ha}$ ammonium nitrate was applied twice through 3rd and 4th irrigations. Befuraline activeingredient herbicide $(60 \% \mathrm{w} / \mathrm{w})$ was applied before sowing, and hoeing was practiced after emergence for weed control.

Harvest was performed through manual pull-out on 15 October 2017 and 20 October 2018. For oil content and fatty acid analyses, $100 \mathrm{~g}$ seeds were sampled from 10 plants of each plot. Seeds were dried to constant moisture before the analyses. 
Table 1. Soil physical characteristics

\begin{tabular}{|l|c|c|c|c|c|c|c|}
\hline Soil depth & Sand (\%) & $\begin{array}{c}\text { Silt } \\
(\%)\end{array}$ & $\begin{array}{c}\text { Clay } \\
(\%)\end{array}$ & Texture & $\begin{array}{c}\text { FC } \\
(\%)\end{array}$ & $\begin{array}{c}\text { PWP } \\
(\%)\end{array}$ & $\begin{array}{c}\text { BD } \\
\left(\mathrm{g} / \mathrm{cm}^{3}\right)\end{array}$ \\
\hline $0-30$ & 60.7 & 24.0 & 15.3 & SL & 16.87 & 7.98 & 1.54 \\
\hline $30-60$ & 95.0 & 2.4 & 2.6 & S & 10.87 & 4.83 & 1.59 \\
\hline $60-90$ & 58.7 & 30.1 & 11.2 & SL & 17.78 & 8.21 & 1.56 \\
\hline
\end{tabular}

Oil extraction and preparation of fatty acid methyl esters (FAME)

Impurities were removed from the seeds, and the cleaned seeds were ground using a ball mill into powder. Lipids were extracted with hexane/isopropanol $(2 \mathrm{v} / \mathrm{v})$ solution overnight in a laboratory-type shaker. The lipid extracts were centrifuged at $10000 \mathrm{~g}$ for $5 \mathrm{~min}$ and filtered after that solvent was removed on a rotary evaporator at $40^{\circ} \mathrm{C}$. After the extraction procedure, fatty acids in the lipid extracts were converted into methyl esters employing 2\% sulphuric acid (v/v) in methanol (Christie, 1990).

\section{Determination of fatty acid composition by GC system}

Lipid fatty acid methyl esters (FAME) in seed samples were prepared using 1-step extraction-trans esterification. The FAME profile for a $0.6-\mu \mathrm{L}$ sample at a split ratio of 1:50 was generated using a gas chromatograph (Schimadzu, GC 2010 plus) equipped with a flame ionization detector (Shimadzu, Kyoto, Japan), a 100-m fused silica capillary column (i.d. $0.25 \mathrm{~mm}$ ) and $\mathrm{H} 2$ as the carrier and fuel gas. The FAME was separated using a temperature gradient program (Chilliard et al., 2013), and the peaks were identified based on comparing retention times with authentic standard (Supelco \#37, Supelco Inc., Bellefonte, PA, USA; L8404 and O5632; Sigma).

Resultant data were subjected to variance analysis. Then, regression analyses were conducted for each fatty acid to assess years, cultivars, irrigation intervals, and irrigation levels visually. In regression analysis, the cubic model was used to see the effects, especially of irrigation levels. Variance analysis and regression graphs were generated with the use of JMP software (SAS Institute, 2014). Finally, biplot graphs were generated with the aid of GGE biplot software to see the effects of different irrigation interval $x$ irrigation level combinations on the fatty acid composition of different cultivars (Yan, 2001).

\section{Results and Discussion}

Initially, oil contents were determined, then unsaturated fatty acids (oleic and linoleic) and saturated fatty acids (palmitic, myristic, arachidic, behenic, and lignoceric) were calculated. Oil contents and fatty acid compositions of peanut cultivars varied with the years, cultivars, irrigation intervals, irrigation levels, and interactions of these factors (Table 2).

Effects of years on total oil, saturated and unsaturated fatty acid contents and fatty acids, except for linoleic acid; effects of cultivars on total oil, saturated and unsaturated fatty acid contents and fatty acids, except for arachidic and behenic acid; effects of irrigation levels on total oil, saturated and unsaturated fatty acid contents and fatty acids, except for arachidic acid; effects of irrigation intervals on total oil, saturated and unsaturated fatty acid contents and fatty acids, except for palmitic, arachidic and behenic acids were found to be highly significant $(\mathrm{P}<0.01)$.

Of double interactions, year $\mathrm{x}$ irrigation interval and year $\mathrm{x}$ irrigation level interactions were found to be significant for total oil content, but insignificant for all fatty acids. Irrigation interval x cultivar interactions were found to be significant for total oil, saturated and unsaturated fatty acid contents, myristic, arachidic and lignoceric acids; cultivar $\mathrm{x}$ irrigation level interactions were found to be significant for total oil, saturated and unsaturated fatty acid contents, myristic, palmitic, behenic, oleic and linoleic acids; irrigation interval $\mathrm{x}$ irrigation level interactions were found to be significant for total oil, saturated and unsaturated fatty acid contents, myristic, oleic and linoleic acids $(\mathrm{P}<0.01)$. 
Of triple interactions, irrigation interval $\mathrm{x}$ cultivar $\mathrm{x}$ irrigation level interactions were found to be significant for total oil, saturated and unsaturated fatty acid contents and all fatty acids, except for linoleic acid; year $\mathrm{x}$ irrigation interval $\mathrm{x}$ irrigation level interactions were found to be significant for total oil contents and myristic acid $(\mathrm{P}<0.01)$.

Table 2. Variance analysis for oil content and fatty acid composition (mean square)

\begin{tabular}{|c|c|c|c|c|c|c|c|c|c|c|c|}
\hline \multirow[b]{2}{*}{ Source } & \multirow[b]{2}{*}{ DF } & \multicolumn{10}{|c|}{ Mean Square } \\
\hline & & $\begin{array}{c}\text { Oil } \\
\text { content }\end{array}$ & Oleic & Linoleic & TUSFA & Myristic & Palmitic & Arachidic & Behenic & Lignocerik & TSFA \\
\hline Year $(Y)$ & 1 & $37.990^{*}$ & $137.910^{* *}$ & 0.535 & $121.2605^{* *}$ & $0.1257^{* *}$ & $10.2022^{* *}$ & $5.3272^{* *}$ & $9.2959^{* *}$ & $4.7724^{* *}$ & $122.9899^{* *}$ \\
\hline Block (Year) & 4 & 4.400 & 3.036 & 2.223 & 0.0744 & 0.0002 & 0.0264 & 0.0672 & 0.0096 & 0.0063 & 0.0426 \\
\hline Cultivar (V) & 2 & $25.460^{*}$ & $429.468^{* *}$ & $287.703^{* *}$ & $14.1843^{* *}$ & $0.0021^{*}$ & $4.3060^{* *}$ & 0.0864 & 0.0804 & $1.8875^{* *}$ & $13.6875^{* *}$ \\
\hline $\mathrm{Y}^{*} \mathrm{C}$ & 2 & $33.920^{*}$ & $122.414^{* *}$ & $116.555^{* *}$ & 0.3316 & $0.0051^{* *}$ & $2.1222^{*}$ & 0.0112 & $0.3704^{*}$ & $0.1742^{* *}$ & 0.4941 \\
\hline Error-1 & 8 & 5.920 & 0.996 & 0.818 & 0.3138 & 0.0004 & 0.3392 & 0.0318 & 0.0507 & 0.0092 & 0.3155 \\
\hline Day (D) & 1 & $6.550^{* *}$ & $10.741^{*}$ & $21.243^{*}$ & $1.7738^{*}$ & $0.0028^{* *}$ & 0.2605 & 0.0249 & 0.0831 & $0.1491^{*}$ & $1.6656^{*}$ \\
\hline $\mathrm{Y}^{*} \mathrm{D}$ & 1 & $83.260^{* *}$ & 4.063 & 2.375 & 0.2253 & 0.0001 & 0.0248 & 0.0003 & 0.0284 & 0.0312 & 0.2741 \\
\hline $\mathrm{D}^{*} \mathrm{C}$ & 2 & $13.540^{* *}$ & 1.797 & 3.456 & 0.2734 & $0.0026^{* *}$ & 0.5492 & $0.3438^{* *}$ & 0.0110 & $0.1079^{*}$ & 0.3311 \\
\hline $\mathrm{Y}^{*} \mathrm{D}^{*} \mathrm{C}$ & 2 & $8.090^{* *}$ & 0.141 & 0.056 & 0.1235 & 0.0001 & 0.0405 & 0.0106 & 0.0003 & 0.0195 & 0.0933 \\
\hline Error-2 & 12 & 0.320 & 2.083 & 2.753 & 0.3535 & 0.0003 & 0.2079 & 0.0253 & 0.0453 & 0.0269 & 0.2944 \\
\hline Irrigation (I) & 3 & $297.240^{* *}$ & $34.922^{* *}$ & $24.199^{* *}$ & $3.3907^{* *}$ & $0.0014^{* *}$ & $1.7928^{* *}$ & 0.0413 & $0.4529^{* *}$ & $0.1515^{*}$ & $3.3826^{* *}$ \\
\hline $\mathrm{Y}^{*} \mathrm{I}$ & 3 & $16.400^{* *}$ & 1.764 & 3.095 & 0.3534 & 0.0005 & 0.1075 & 0.0057 & 0.0199 & 0.0097 & 0.2997 \\
\hline $\mathrm{V}^{*} \mathrm{I}$ & 6 & $23.130^{* *}$ & $11.163^{* *}$ & $9.635^{* *}$ & 0.7552 & $0.0044^{* *}$ & $0.7752^{*}$ & 0.0259 & $0.1418^{*}$ & 0.0559 & 0.8141 \\
\hline $\mathrm{Y}^{*} \mathrm{C}^{*} \mathrm{I}$ & 6 & $11.510^{* *}$ & 2.440 & 2.185 & 0.1388 & 0.0009 & 0.0471 & 0.0125 & 0.0231 & 0.0113 & 0.0954 \\
\hline $\mathrm{D}^{*} \mathrm{I}$ & 3 & $16.430^{* *}$ & $12.666^{* *}$ & $11.392^{*}$ & 0.3241 & $0.0066^{* *}$ & 0.3732 & 0.0704 & 0.0326 & 0.0261 & 0.3832 \\
\hline $\mathrm{Y}^{*} \mathrm{D}^{*} \mathrm{I}$ & 3 & $32.330^{* *}$ & 1.325 & 1.551 & 0.0417 & $0.0016^{* *}$ & 0.0531 & 0.0322 & 0.0086 & 0.0052 & 0.0420 \\
\hline $\mathrm{D}^{*} \mathrm{C}^{*} \mathrm{I}$ & 6 & $9.340^{* *}$ & $9.888^{* *}$ & 4.357 & $4.1174^{* *}$ & $0.0044^{* *}$ & $0.9680^{* *}$ & $0.1173^{*}$ & $0.3788^{* *}$ & $0.2034^{* *}$ & $4.2432^{* *}$ \\
\hline $\mathrm{Y}^{*} \mathrm{D}^{*} \mathrm{C}^{*} \mathrm{I}$ & 6 & $30.770^{* *}$ & 0.534 & 0.312 & 0.3118 & $0.0009^{*}$ & 0.0843 & 0.0264 & 0.0256 & 0.0163 & 0.2288 \\
\hline Error-3 & 72 & 1.908 & 3.652 & 3.584 & 0.5388 & 0.0004 & 0.2933 & 0.0432 & 0.0655 & 0.0468 & 0.5277 \\
\hline $\begin{array}{l}\text { Coefficient } \\
\text { of variation }\end{array}$ & & 3.23 & 3.94 & 5.91 & 0.91 & 11.70 & 5.00 & 6.36 & 7.28 & 13.13 & 3.74 \\
\hline R-Square & & 0.96 & 0.86 & 0.81 & 0.84 & 0.90 & 0.69 & 0.73 & 0.78 & 0.78 & 0.84 \\
\hline
\end{tabular}

DF: Degrees of freedom, TUSFA: Total unsaturated fatty acid, TSFA: Total saturated fatty acid ${ }^{* *}: \mathrm{P}<0.01,{ }^{*}: \mathrm{P}<0.05$

Since the effects of the years on oil content and fatty acid compositions were found to be significant, assessments were made separately for fatty acids through distributing the effects of cultivars, irrigation intervals over the year. Resultant averages are provided in Tables 3 and 4; regression graphs are presented in Figures 1,2, and 3; biplot graphs are presented in Figure 4. 
Akçura S et al. (2021). Not Bot Horti Agrobo 49(2):12224
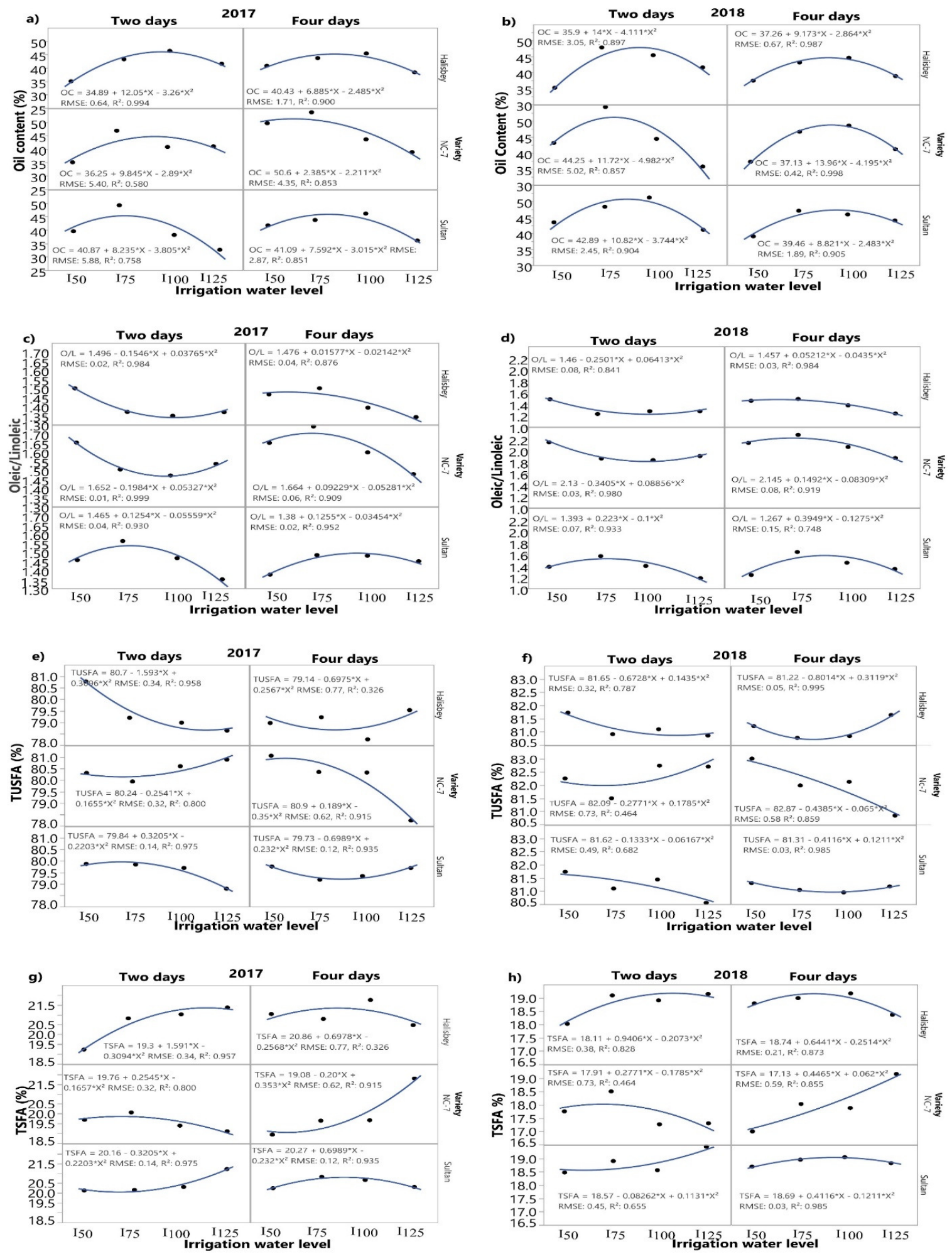

Figure 1. Change in a-b) oil content, c-d) oleic/linoleic acid ratio, e-f) total unsaturated fatty acids, g-h) total saturated fatty acids based on year, cultivar, irrigation interval, and irrigation level 
Akçura S et al. (2021). Not Bot Horti Agrobo 49(2):12224


Figure 2. Change in a-b) oleic, c-d) linoleic and e-f) palmitic acid contents based on year, cultivar, irrigation interval, and irrigation level 
Akçura S et al. (2021). Not Bot Horti Agrobo 49(2):12224
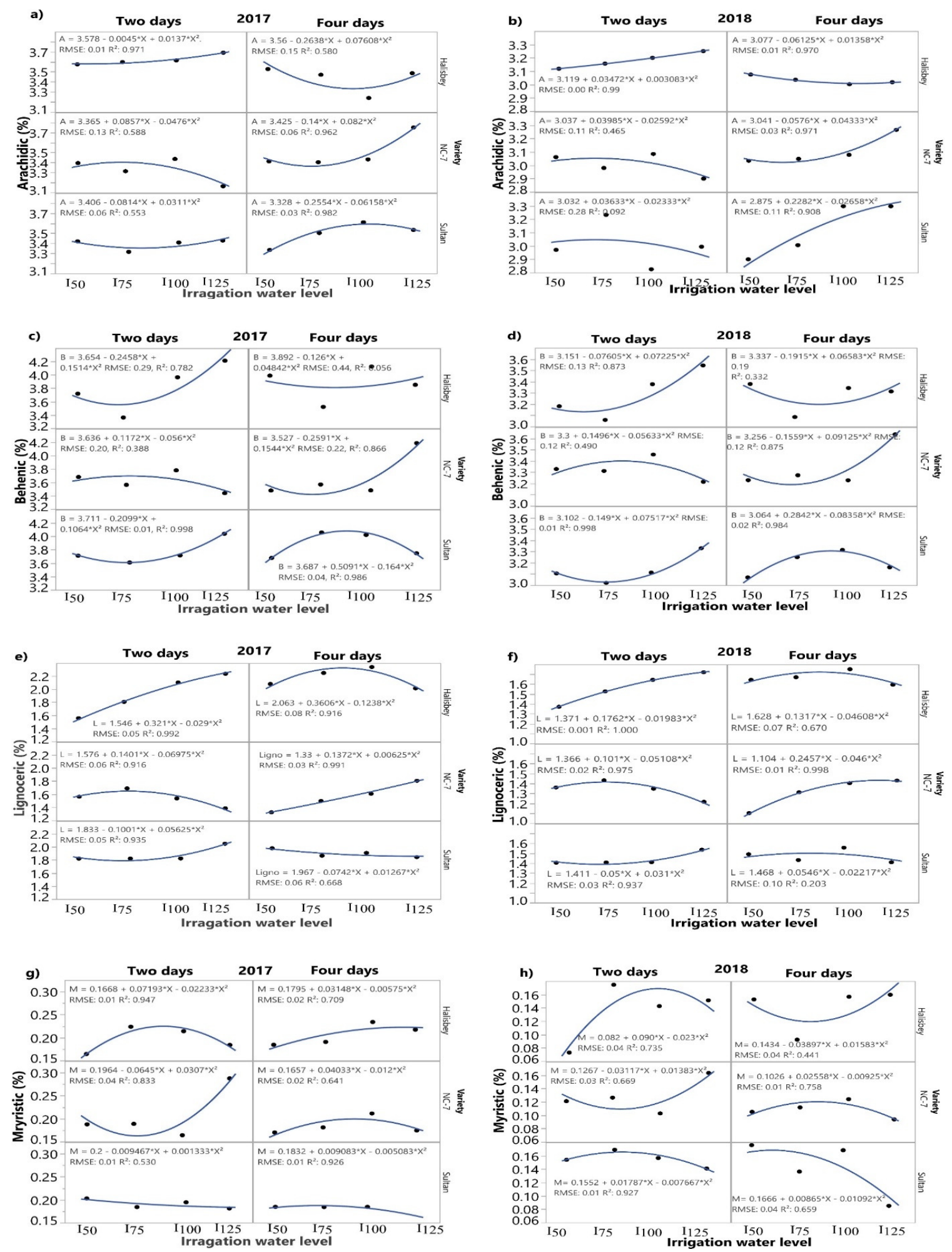

Figure 3. Change in $a-b$ ) arachidic, $c-d$ ) behenic, e-f) lignoceric, g-h) myristic acid contents based on year, cultivar, irrigation interval, and irrigation level 
Akçura S et al. (2021). Not Bot Horti Agrobo 49(2):12224
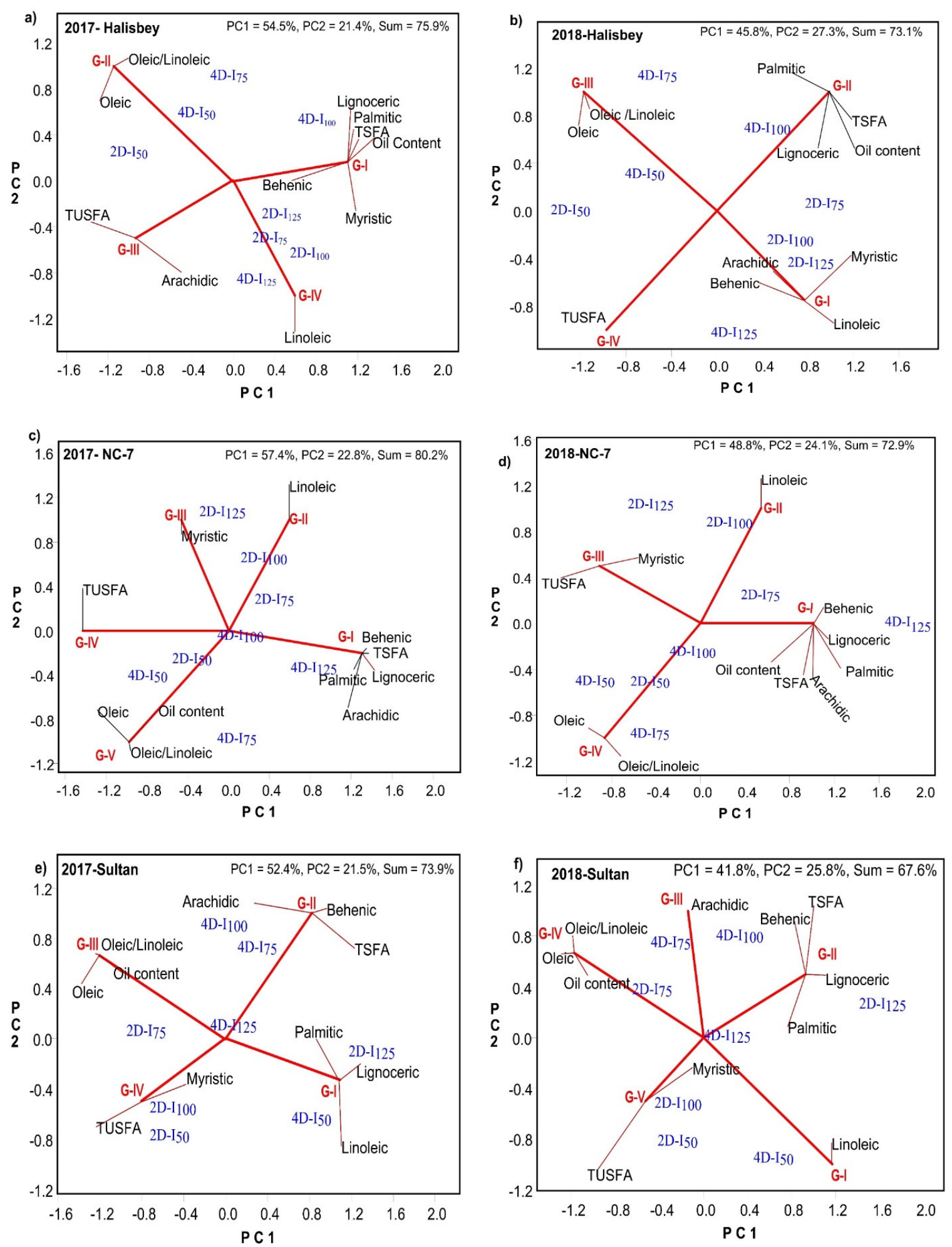

Figure 4. Relationships between investigated parameters of the years and attribute groups 
Total oil, saturated, and unsaturated fatty acid contents of peanut cultivars were significantly influenced by the years and irrigation treatments. Effects of irrigation intervals and irrigation levels on total saturated and unsaturated fatty acid contents significantly varied with the cultivars. Generally, the saturated fatty acid ratio increased, and the unsaturated fatty acid ratio decreased (Figure 1e-h). In general, the total oil ratio at a twoday irrigation interval was lower in the first year (40.8\%) than in the second year (43.6\%). In the four-day irrigation interval, the oil ratio of the second year $(42.8 \%)$ was lower than the oil ratio of the first year $(43.5 \%)$. As can be seen in regression analyses, peak levels were determined for the greatest oil ratios at two and four-day irrigations of both years (Figure 1a and $\mathrm{b}$ ). The lowest oil ratios of all cultivars were obtained from $\mathrm{I}_{50}$ and $\mathrm{I}_{125}$ irrigation levels. Since the soil texture of the experimental site was sandy, plants were highly influenced by water stress in deficit irrigation treatment and negatively influenced by excessive water in $\mathrm{I}_{125}$ treatments. The greatest oil ratios were obtained from the $\mathrm{I}_{75}$ irrigation level. In general, at both irrigation intervals, oil ratios significantly increased from $\mathrm{I}_{50}$ to $\mathrm{I}_{75}$ level, but a significant change was observed at the $\mathrm{I}_{100}$ level in 'Halisbey' and 'Sultan' cultivars. On the other hand, 'NC-7' exhibited a different response to $\mathrm{I}_{100}$ treatment from the other two cultivars (Figure 1a and b). Aydınşakir et al. (2019) reported the greatest crude oil ratio of 'NC-7' cultivar under clay-loam soil conditions at $\mathrm{I}_{100}$ and $\mathrm{I}_{125}$ irrigation levels.

Considering the entire factors of the years together, it was observed that the values of oleic acid and total unsaturated fatty acids were greater in the first year than the second year and a reverse case was valid for the total oil ratio, saturated fatty acid ratio and the other fatty acids (Tables 3 and 4).

Table 3. Averages for total oil content and fatty acid contents based on irrigation interval, irrigation level and cultivar in 2017

\begin{tabular}{|c|c|c|c|c|c|c|c|c|c|c|}
\hline \multirow{2}{*}{ Irrigation } & \multirow{2}{*}{ Oil Content } & \multicolumn{3}{|c|}{ Unsaturated fatty acids } & \multicolumn{6}{|c|}{ Saturated fatty acids } \\
\hline & & Oleic & Linoleic & TUSFA & Palmitic & Myristic & Arachidic & Behenic & Lignoceric & TSFA \\
\hline \multicolumn{11}{|c|}{ Two days } \\
\hline \multicolumn{11}{|c|}{ 'Halisbey' } \\
\hline$I_{5_{0}}$ & $35.0 \pm 1.7$ & $48.74 \pm 1.1$ & $32.99 \pm 1.1$ & $81.72 \pm 0.6$ & $10.28 \pm 0.4$ & $0.07 \pm 0.01$ & $3.12 \pm 0.05$ & $3.18 \pm 0.18$ & $1.37 \pm 0.09$ & $18.02 \pm 0.44$ \\
\hline$I_{75}$ & $43.3 \pm 1.0$ & $44.48 \pm 0.7$ & $36.43 \pm 0.7$ & $80.91 \pm 0.1$ & $11.17 \pm 0.4$ & $0.17 \pm 0.03$ & $3.16 \pm 0.13$ & $3.06 \pm 0.05$ & $1.53 \pm 0.22$ & $19.09 \pm 0.10$ \\
\hline$I_{100}$ & $46.4 \pm 1.8$ & $45.36 \pm 2.2$ & $35.74 \pm 2.1$ & $81.09 \pm 0.2$ & $10.54 \pm 0.6$ & $0.14 \pm 0.02$ & $3.2 \pm 0.13$ & $3.38 \pm 0.25$ & $1.64 \pm 0.12$ & $18.91 \pm 0.17$ \\
\hline$I_{125}$ & $41.5 \pm 0.9$ & $45.22 \pm 3.5$ & $35.63 \pm 3.8$ & $80.85 \pm 0.6$ & $10.48 \pm 0.6$ & $0.15 \pm 0.03$ & $3.25 \pm 0.09$ & $3.54 \pm 0.05$ & $1.72 \pm 0.05$ & $19.15 \pm 0.61$ \\
\hline Mean & 41.6 & 45.95 & 35.2 & 81.15 & 10.62 & 0.14 & 3.18 & 3.29 & 1.57 & 18.79 \\
\hline \multicolumn{11}{|c|}{ 'NC-7' } \\
\hline$I_{50}$ & $35.0 \pm 0.9$ & $56.03 \pm 1.1$ & $26.22 \pm 1.7$ & $82.25 \pm 1.0$ & $9.88 \pm 0.7$ & $0.12 \pm 0.01$ & $3.06 \pm 0.18$ & $3.33 \pm 0.22$ & $1.36 \pm 0.19$ & $17.75 \pm 0.96$ \\
\hline$I_{75}$ & $46.8 \pm 0.6$ & $52.95 \pm 1.5$ & $28.55 \pm 0.5$ & $81.50 \pm 1.4$ & $10.65 \pm 0.7$ & $0.13 \pm 0.01$ & $2.98 \pm 0.16$ & $3.31 \pm 0.28$ & $1.43 \pm 0.25$ & $18.5 \pm 1.36$ \\
\hline $\mathrm{I}_{100}$ & $40.8 \pm 1.0$ & $53.46 \pm 0.5$ & $29.27 \pm 0.3$ & $82.74 \pm 0.2$ & $9.27 \pm 0.5$ & $0.10 \pm 0.02$ & $3.08 \pm 0.18$ & $3.46 \pm 0.21$ & $1.35 \pm 0.09$ & $17.26 \pm 0.23$ \\
\hline$I_{125}$ & $41.0 \pm 0.3$ & $54.16 \pm 1.3$ & $28.54 \pm 0.6$ & $82.70 \pm 0.8$ & $9.81 \pm 0.6$ & $0.16 \pm 0.01$ & $2.9 \pm 0.09$ & $3.21 \pm 0.14$ & $1.21 \pm 0.04$ & $17.3 \pm 0.77$ \\
\hline Mean & 40.9 & 54.15 & 28.15 & 82.3 & 9.9 & 0.13 & 3.01 & 3.33 & 1.34 & 17.7 \\
\hline \multicolumn{11}{|c|}{ 'Sultan' } \\
\hline$I_{50}$ & $39.6 \pm 0.7$ & $47.35 \pm 1.2$ & $34.39 \pm 1.0$ & $81.73 \pm 0.5$ & $10.84 \pm 0.4$ & $0.15 \pm 0.04$ & $2.97 \pm 0.05$ & $3.1 \pm 0.27$ & $1.4 \pm 0.19$ & $18.47 \pm 0.55$ \\
\hline $\mathrm{I}_{75}$ & $49.2 \pm 1.6$ & $49.46 \pm 3.0$ & $31.64 \pm 2.8$ & $81.10 \pm 0.2$ & $11.07 \pm 0.3$ & $0.17 \pm 0.01$ & $3.23 \pm 0.10$ & $3.02 \pm 0.21$ & $1.41 \pm 0.10$ & $18.90 \pm 0.16$ \\
\hline $\mathrm{I}_{100}$ & $38.2 \pm 2.1$ & $47.38 \pm 0.7$ & $34.06 \pm 0.5$ & $81.44 \pm 0.8$ & $11.06 \pm 0.4$ & $0.16 \pm 0.03$ & $2.82 \pm 0.38$ & $3.11 \pm 0.06$ & $1.41 \pm 0.08$ & $18.56 \pm 0.79$ \\
\hline$I_{125}$ & $32.6 \pm 0.3$ & $43.56 \pm 1.1$ & $36.99 \pm 1.6$ & $80.56 \pm 0.6$ & $11.44 \pm 0.3$ & $0.14 \pm 0.01$ & $2.99 \pm 0.06$ & $3.33 \pm 0.10$ & $1.54 \pm 0.11$ & $19.44 \pm 0.58$ \\
\hline Mean & 39.9 & 46.94 & 34.27 & 81.21 & 11.1 & 0.16 & 3.01 & 3.14 & 1.44 & 18.84 \\
\hline D mean & 40.8 & 49.01 & 32.54 & 81.55 & 10.54 & 0.14 & 3.07 & 3.25 & 1.45 & 18.44 \\
\hline \multicolumn{11}{|c|}{ Four days } \\
\hline \multicolumn{11}{|c|}{ 'Halisbey" } \\
\hline$I_{50}$ & $40.8 \pm 0.2$ & $48.08 \pm 1.7$ & $33.13 \pm 1.5$ & $81.21 \pm 0.6$ & $10.54 \pm 0.4$ & $0.15 \pm 0.02$ & $3.08 \pm 0.12$ & $3.38 \pm 0.16$ & $1.64 \pm 0.15$ & $18.79 \pm 0.63$ \\
\hline$I_{75}$ & $43.7 \pm 1.3$ & $48.23 \pm 0.6$ & $32.53 \pm 1.4$ & $80.77 \pm 1.2$ & $11.11 \pm 0.5$ & $0.09 \pm 0.01$ & $3.04 \pm 0.28$ & $3.09 \pm 0.15$ & $1.67 \pm 0.16$ & $18.99 \pm 0.79$ \\
\hline $\mathrm{I}_{100}$ & $45.4 \pm 1.7$ & $46.73 \pm 2.5$ & $34.10 \pm 3.4$ & $80.83 \pm 1.1$ & $10.91 \pm 0.9$ & $0.16 \pm 0.03$ & $3.00 \pm 0.29$ & $3.34 \pm 0.24$ & $1.75 \pm 0.24$ & $19.17 \pm 1.06$ \\
\hline$I_{125}$ & $38.3 \pm 2.4$ & $44.99 \pm 2.1$ & $36.65 \pm 2.9$ & $81.64 \pm 0.8$ & $10.28 \pm 0.7$ & $0.16 \pm 0.01$ & $3.02 \pm 0.03$ & $3.31 \pm 0.13$ & $1.59 \pm 0.02$ & $18.36 \pm 0.82$ \\
\hline Mean & 42.1 & 47.01 & 34.1 & 81.11 & 10.71 & 0.14 & 3.03 & 3.28 & 1.66 & 18.83 \\
\hline \multicolumn{11}{|c|}{ 'NC-7' } \\
\hline$I_{50}$ & $49.6 \pm 1.7$ & $56.46 \pm 2.2$ & $26.55 \pm 2.5$ & $83.00 \pm 0.6$ & $9.53 \pm 0.5$ & $0.11 \pm 0.02$ & $3.03 \pm 0.18$ & $3.23 \pm 0.18$ & $1.10 \pm 0.26$ & $17.00 \pm 0.61$ \\
\hline$I_{75}$ & $53.7 \pm 0.7$ & $56.89 \pm 0.9$ & $25.10 \pm 0.4$ & $81.98 \pm 0.5$ & $10.28 \pm 0.7$ & $0.11 \pm 0.02$ & $3.05 \pm 0.11$ & $3.27 \pm 0.17$ & $1.31 \pm 0.13$ & $18.03 \pm 0.5$ \\
\hline$I_{100}$ & $43.6 \pm 0.5$ & $55.25 \pm 3.5$ & $26.88 \pm 3.0$ & $82.12 \pm 0.7$ & $10.04 \pm 0.7$ & $0.12 \pm 0.01$ & $3.08 \pm 0.17$ & $3.23 \pm 0.22$ & $1.40 \pm 0.13$ & $17.88 \pm 0.65$ \\
\hline$I_{125}$ & $38.8 \pm 0.3$ & $52.61 \pm 2.3$ & $28.23 \pm 2.8$ & $80.84 \pm 0.8$ & $10.73 \pm 0.5$ & $0.09 \pm 0.01$ & $3.27 \pm 0.18$ & $3.64 \pm 0.09$ & $1.43 \pm 0.1$ & $19.16 \pm 0.83$ \\
\hline Mean & 46.4 & 55.3 & 26.69 & 81.99 & 10.15 & 0.11 & 3.11 & 3.34 & 1.31 & 18.01 \\
\hline \multicolumn{11}{|c|}{ 'Sultan' } \\
\hline$I_{50}$ & $41.7 \pm 1.3$ & $44.90 \pm 4.8$ & $36.40 \pm 4.4$ & $81.30 \pm 0.4$ & $11.06 \pm 0.9$ & $0.18 \pm 0.01$ & $2.90 \pm 0.32$ & $3.07 \pm 0.21$ & $1.49 \pm 0.04$ & $18.70 \pm 0.43$ \\
\hline$I_{75}$ & $43.7 \pm 0.5$ & $50.28 \pm 1.0$ & $30.77 \pm 0.8$ & $81.04 \pm 0.2$ & $11.13 \pm 0.3$ & $0.14 \pm 0.03$ & $3.01 \pm 0.08$ & $3.25 \pm 0.21$ & $1.43 \pm 0.10$ & $18.96 \pm 0.21$ \\
\hline$I_{100}$ & $46.1 \pm 0.8$ & $47.87 \pm 2.9$ & $33.09 \pm 3.0$ & $80.95 \pm 0.5$ & $10.71 \pm 0.7$ & $0.17 \pm 0.01$ & $3.30 \pm 0.31$ & $3.31 \pm 0.05$ & $1.56 \pm 0.34$ & $19.05 \pm 0.54$ \\
\hline$I_{125}$ & $36.1 \pm 2.3$ & $46.44 \pm 3.6$ & $34.73 \pm 3.4$ & $81.17 \pm 0.8$ & $10.88 \pm 0.4$ & $0.09 \pm 0.02$ & $3.30 \pm 0.21$ & $3.16 \pm 0.1$ & $1.41 \pm 0.13$ & $18.83 \pm 0.78$ \\
\hline Mean & 41.9 & 47.37 & 33.75 & 81.12 & 10.95 & 0.14 & 3.12 & 3.2 & 1.47 & 18.88 \\
\hline D.mean & 43.5 & 49.89 & 31.51 & 81.41 & 10.6 & 0.13 & 3.27 & 1.48 & 1.48 & 18.57 \\
\hline G. Mean & 42.2 & 49.45 & 32.03 & 81.48 & 10.57 & 0.14 & 3.08 & 3.26 & 1.47 & 18.51 \\
\hline
\end{tabular}


Table 4. Averages for total oil content and fatty acid contents based on irrigation interval, irrigation level and cultivar in 2018

\begin{tabular}{|c|c|c|c|c|c|c|c|c|c|c|}
\hline \multirow{2}{*}{ Irrigation } & \multirow{2}{*}{ Oil Content } & \multicolumn{3}{|c|}{ Unsaturated fatty acids } & \multicolumn{6}{|c|}{ Saturated fatty acids } \\
\hline & & Oleic & Linoleic & TUSFA & Palmitic & Myristic & Arachidic & Behenic & Lignoceric & TSFA \\
\hline \multicolumn{11}{|c|}{ Two days } \\
\hline \multicolumn{11}{|c|}{ 'Halisbey' } \\
\hline$I_{50}$ & $35.2 \pm 1.8$ & $48.74 \pm 1.1$ & $32.99 \pm 1.1$ & $81.72 \pm 0.6$ & $10.28 \pm 0.4$ & $0.07 \pm 0.01$ & $3.12 \pm 0.05$ & $3.18 \pm 0.18$ & $1.37 \pm 0.09$ & $18.02 \pm 0.44$ \\
\hline$I_{75}$ & $47.8 \pm 1.5$ & $44.48 \pm 0.7$ & $36.43 \pm 0.7$ & $80.91 \pm 0.1$ & $11.17 \pm 0.4$ & $0.17 \pm 0.03$ & $3.16 \pm 0.13$ & $3.06 \pm 0.05$ & $1.53 \pm 0.22$ & $19.09 \pm 0.10$ \\
\hline$I_{100}$ & $45.4 \pm 1.0$ & $45.36 \pm 2.2$ & $35.74 \pm 2.1$ & $81.09 \pm 0.2$ & $10.54 \pm 0.6$ & $0.14 \pm 0.02$ & $3.2 \pm 0.13$ & $3.38 \pm 0.25$ & $1.64 \pm 0.12$ & $18.91 \pm 0.17$ \\
\hline$I_{125}$ & $41.6 \pm 1.2$ & $45.22 \pm 3.5$ & $35.63 \pm 3.8$ & $80.85 \pm 0.6$ & $10.48 \pm 0.6$ & $0.15 \pm 0.03$ & $3.25 \pm 0.09$ & $3.54 \pm 0.05$ & $1.72 \pm 0.05$ & $19.15 \pm 0.61$ \\
\hline Mean & 42.5 & 45.95 & 35.2 & 81.15 & 10.62 & 0.14 & 3.18 & 3.29 & 1.57 & 18.79 \\
\hline \multicolumn{11}{|c|}{ 'NC-7' } \\
\hline$I_{50}$ & $43.1 \pm 1.9$ & $56.03 \pm 1.1$ & $26.22 \pm 1.7$ & $82.25 \pm 1.0$ & $9.88 \pm 0.7$ & $0.12 \pm 0.01$ & $3.06 \pm 0.18$ & $3.33 \pm 0.22$ & $1.36 \pm 0.19$ & $17.75 \pm 0.96$ \\
\hline$I_{75}$ & $54.4 \pm 1.4$ & $52.95 \pm 1.5$ & $28.55 \pm 0.5$ & $81.50 \pm 1.4$ & $10.65 \pm 0.7$ & $0.13 \pm 0.01$ & $2.98 \pm 0.16$ & $3.31 \pm 0.28$ & $1.43 \pm 0.25$ & $18.5 \pm 1.36$ \\
\hline$I_{100}$ & $44.4 \pm 1.6$ & $53.46 \pm 0.5$ & $29.27 \pm 0.3$ & $82.74 \pm 0.2$ & $9.27 \pm 0.5$ & $0.10 \pm 0.02$ & $3.08 \pm 0.18$ & $3.46 \pm 0.21$ & $1.35 \pm 0.09$ & $17.26 \pm 0.23$ \\
\hline$I_{125}$ & $35.7 \pm 1.4$ & $54.16 \pm 1.3$ & $28.54 \pm 0.6$ & $82.70 \pm 0.8$ & $9.81 \pm 0.6$ & $0.16 \pm 0.01$ & $2.9 \pm 0.09$ & $3.21 \pm 0.14$ & $1.21 \pm 0.04$ & $17.3 \pm 0.77$ \\
\hline Mean & 44.4 & 54.15 & 28.15 & 82.3 & 9.9 & 0.13 & 3.01 & 3.33 & 1.34 & 17.7 \\
\hline \multicolumn{11}{|c|}{ 'Sultan' } \\
\hline $\mathbf{I}_{\mathbf{5 0}}$ & $43.4 \pm 3.3$ & $47.35 \pm 1.2$ & $34.39 \pm 1.0$ & $81.73 \pm 0.5$ & $10.84 \pm 0.4$ & $0.15 \pm 0.04$ & $2.97 \pm 0.05$ & $3.1 \pm 0.27$ & $1.4 \pm 0.19$ & $18.47 \pm 0.55$ \\
\hline$I_{75}$ & $48.3 \pm 1.9$ & $49.46 \pm 3.0$ & $31.64 \pm 2.8$ & $81.10 \pm 0.2$ & $11.07 \pm 0.3$ & $0.17 \pm 0.01$ & $3.23 \pm 0.10$ & $3.02 \pm 0.21$ & $1.41 \pm 0.10$ & $18.90 \pm 0.16$ \\
\hline$I_{100}$ & $51.2 \pm 0.4$ & $47.38 \pm 0.7$ & $34.06 \pm 0.5$ & $81.44 \pm 0.8$ & $11.06 \pm 0.4$ & $0.16 \pm 0.03$ & $2.82 \pm 0.38$ & $3.11 \pm 0.06$ & $1.41 \pm 0.08$ & $18.56 \pm 0.79$ \\
\hline$I_{125}$ & $41.1 \pm 1.3$ & $43.56 \pm 1.1$ & $36.99 \pm 1.6$ & $80.56 \pm 0.6$ & $11.44 \pm 0.3$ & $0.14 \pm 0.01$ & $2.99 \pm 0.06$ & $3.33 \pm 0.10$ & $1.54 \pm 0.11$ & $19.44 \pm 0.58$ \\
\hline Mean & 46.0 & 46.94 & 34.27 & 81.21 & 11.1 & 0.16 & 3.01 & 3.14 & 1.44 & 18.84 \\
\hline D. mean & 44.3 & 49.01 & 32.54 & 81.55 & 10.54 & 0.14 & 3.07 & 3.25 & 1.45 & 18.44 \\
\hline \multicolumn{11}{|c|}{ Four days } \\
\hline \multicolumn{11}{|c|}{ 'Halisbey' } \\
\hline $\mathbf{I}_{\mathbf{5} 0}$ & $37.4 \pm 1.9$ & $48.08 \pm 1.7$ & $33.13 \pm 1.5$ & $81.21 \pm 0.6$ & $10.54 \pm 0.4$ & $0.15 \pm 0.02$ & $3.08 \pm 0.12$ & $3.38 \pm 0.16$ & $1.64 \pm 0.15$ & $18.79 \pm 0.63$ \\
\hline$I_{75}$ & $43.1 \pm 1.1$ & $48.23 \pm 0.6$ & $32.53 \pm 1.4$ & $80.77 \pm 1.2$ & $11.11 \pm 0.5$ & $0.09 \pm 0.01$ & $3.04 \pm 0.28$ & $3.09 \pm 0.15$ & $1.67 \pm 0.16$ & $18.99 \pm 0.79$ \\
\hline$I_{100}$ & $44.6 \pm 1.1$ & $46.73 \pm 2.5$ & $34.10 \pm 3.4$ & $80.83 \pm 1.1$ & $10.91 \pm 0.9$ & $0.16 \pm 0.03$ & $3.00 \pm 0.29$ & $3.34 \pm 0.24$ & $1.75 \pm 0.24$ & $19.17 \pm 1.06$ \\
\hline$I_{125}$ & $38.9 \pm 2.2$ & $44.99 \pm 2.1$ & $36.65 \pm 2.9$ & $81.64 \pm 0.8$ & $10.28 \pm 0.7$ & $0.16 \pm 0.01$ & $3.02 \pm 0.03$ & $3.31 \pm 0.13$ & $1.59 \pm 0.02$ & $18.36 \pm 0.82$ \\
\hline Mean & 41.0 & 47.01 & 34.1 & 81.11 & 10.71 & 0.14 & 3.03 & 3.28 & 1.66 & 18.83 \\
\hline \multicolumn{11}{|c|}{ 'NC-7' } \\
\hline$I_{50}$ & $37.2 \pm 2.1$ & $56.46 \pm 2.2$ & $26.55 \pm 2.5$ & $83.00 \pm 0.6$ & $9.53 \pm 0.5$ & $0.11 \pm 0.02$ & $3.03 \pm 0.18$ & $3.23 \pm 0.18$ & $1.10 \pm 0.26$ & $17.00 \pm 0.61$ \\
\hline $\mathbf{I}_{75}$ & $46.6 \pm 4.4$ & $56.89 \pm 0.9$ & $25.10 \pm 0.4$ & $81.98 \pm 0.5$ & $10.28 \pm 0.7$ & $0.11 \pm 0.02$ & $3.05 \pm 0.11$ & $3.27 \pm 0.17$ & $1.31 \pm 0.13$ & $18.03 \pm 0.5$ \\
\hline$I_{100}$ & $48.5 \pm 3.0$ & $55.25 \pm 3.5$ & $26.88 \pm 3.0$ & $82.12 \pm 0.7$ & $10.04 \pm 0.7$ & $0.12 \pm 0.01$ & $3.08 \pm 0.17$ & $3.23 \pm 0.22$ & $1.40 \pm 0.13$ & $17.88 \pm 0.65$ \\
\hline$I_{125}$ & $41.1 \pm 1.1$ & $52.61 \pm 2.3$ & $28.23 \pm 2.8$ & $80.84 \pm 0.8$ & $10.73 \pm 0.5$ & $0.09 \pm 0.01$ & $3.27 \pm 0.18$ & $3.64 \pm 0.09$ & $1.43 \pm 0.1$ & $19.16 \pm 0.83$ \\
\hline Mean & 43.4 & 55.3 & 26.69 & 81.99 & 10.15 & 0.11 & 3.11 & 3.34 & 1.31 & 18.01 \\
\hline \multicolumn{11}{|c|}{ 'Sultan' } \\
\hline $\mathbf{I}_{50}$ & $39.0 \pm 0.7$ & $44.90 \pm 4.8$ & $36.40 \pm 4.4$ & $81.30 \pm 0.4$ & $11.06 \pm 0.9$ & $0.18 \pm 0.01$ & $2.90 \pm 0.32$ & $3.07 \pm 0.21$ & $1.49 \pm 0.04$ & $18.70 \pm 0.43$ \\
\hline $\mathbf{I}_{75}$ & $47.1 \pm 1.4$ & $50.28 \pm 1.0$ & $30.77 \pm 0.8$ & $81.04 \pm 0.2$ & $11.13 \pm 0.3$ & $0.14 \pm 0.03$ & $3.01 \pm 0.08$ & $3.25 \pm 0.21$ & $1.43 \pm 0.10$ & $18.96 \pm 0.21$ \\
\hline$I_{100}$ & $45.9 \pm 1.5$ & $47.87 \pm 2.9$ & $33.09 \pm 3.0$ & $80.95 \pm 0.5$ & $10.71 \pm 0.7$ & $0.17 \pm 0.01$ & $3.30 \pm 0.31$ & $3.31 \pm 0.05$ & $1.56 \pm 0.34$ & $19.05 \pm 0.54$ \\
\hline$I_{125}$ & $44.0 \pm 3.1$ & $46.44 \pm 3.6$ & $34.73 \pm 3.4$ & $81.17 \pm 0.8$ & $10.88 \pm 0.4$ & $0.09 \pm 0.02$ & $3.30 \pm 0.21$ & $3.16 \pm 0.1$ & $1.41 \pm 0.13$ & $18.83 \pm 0.78$ \\
\hline Mean & 44 & 47.37 & 33.75 & 81.12 & 10.95 & 0.14 & 3.12 & 3.2 & 1.47 & 18.88 \\
\hline D.mean & 42.8 & 49.89 & 31.51 & 81.41 & 10.6 & 0.13 & 3.27 & 1.48 & 1.48 & 18.57 \\
\hline G.Mean & 43.6 & 49.45 & 32.03 & 81.48 & 10.57 & 0.14 & 3.08 & 3.26 & 1.47 & 18.51 \\
\hline
\end{tabular}

The major unsaturated fatty acids of peanuts were reported as oleic and linoleic acids, and the major saturated fatty acid was reported as palmitic acid (Janila et al., 2016). Therefore, these fatty acids were separately assessed from the others, and regression graphs, including years, irrigation intervals, cultivars, and irrigation levels, are presented in Figure 2.

As can be seen in Figure 2, while regression curves of oleic acid concentrations were similar for the years and irrigation intervals, they were significantly different for cultivars and irrigation levels.

In 'Halisbey' cultivar, the greatest oleic acid content was obtained from the two-day interval of $\mathrm{I}_{50}$ irrigation level (48.74\% in 2017 and $48.46 \%$ in 2018), and the lowest value was obtained from $\mathrm{I}_{125}$ irrigation level (45.42\% in 2017 and 45.22\% in 2018). In the two-day irrigation interval of 'Halisbey' cultivar, changes in oleic acid contents were similar in $\mathrm{I}_{75}$ and $\mathrm{I}_{100}$ treatments (Figure $2 \mathrm{a}$ ). In the four-day irrigation interval of 'Halisbey' cultivar, oleic acid content was $46.39 \%$ in 2017 and $47.01 \%$ in 2018. As it was in the two-day irrigation interval, the greatest oleic acid content was observed in $\mathrm{I}_{50}$ treatment, and the lowest oleic acid content was observed in $\mathrm{I}_{125}$ treatments of both years (Figure 2a and $\mathrm{b}$ ).

In a two-day irrigation interval of NC-7 cultivar, the greatest oleic acid content was measured as $50.04 \%$ in 2017 and 56.03\% in 2018. Despite this difference, regression curves were quite similar in both years (Figure $2 \mathrm{a}$ and $\mathrm{b}$ ). The lowest oleic contents were observed in $\mathrm{I}_{75}$ and $\mathrm{I}_{100}$ irrigation levels of both years, and slightly greater oleic acid contents were observed in the $\mathrm{I}_{125}$ irrigation level than the previous two doses. In the four-day irrigation interval of NC-7 cultivar, the greatest oleic acid contents were observed in $\mathrm{I}_{75}$ and $\mathrm{I}_{50}$ irrigation levels ( 51.05 and $50.49 \%$ in 2017 and 56.89 and $56.46 \%$ in 2018). The lowest oleic acid contents in this irrigation interval were observed in the $\mathrm{I}_{125}$ irrigation level (46.67\% in 2016 and 52.61\% in 2018) (Tables 3 and 4).

In a two-day irrigation interval of 'Sultan' cultivar, the greatest oleic acid contents were obtained from the $\mathrm{I}_{75}$ irrigation level (48.68\% in 2017 and $49.46 \%$ in 2018), and the lowest oleic acid contents were obtained 
from $I_{125}$ treatments ( $45.26 \%$ in 2017 and $43.56 \%$ in 2018). In a two-day irrigation interval of 'Sultan' cultivar, years had slight effects on oleic acid contents. Consequently, resultant regression curves were quite similar to each other (Figure $2 \mathrm{a}$ and b). In a four-day irrigation interval of 'Sultan' cultivar, oleic acid contents of 2017 varied between $46.19-47.35 \%$ based on irrigation levels. In 2018, the greatest oleic content was obtained from $\mathrm{I}_{75}(50.28 \%)$, and the lowest oleic acid content was obtained from $\mathrm{I}_{50}(44.90 \%)$ treatment (Table 3 and 4$)$. The regression curves generated based on irrigation intervals, and irrigation levels of 'Sultan' cultivar were significantly different in 2017 and 2018 (Figure $2 a$ and b).

In previous studies, oleic acid contents of peanuts were reported as between $35-72 \%$ and such differences were attributed to cultivars and environmental factors (Liu et al., 2008; Abbas et al., 2 018). Significant effects of irrigation levels and drought treatments on oleic acid contents were also reported (Dwivedi et al., 1996).

For linoleic acid contents, in a two-day irrigation interval, 'Halisbey' and 'NC-7' cultivars exhibited similar responses to irrigation levels in 2017 and 2018 (Figure 2c and d). In two-day irrigation interval of 2017, the lowest linoleic acid contents of these two cultivars were obtained from $\mathrm{I}_{50}$ irrigation level $(32.32 \%$ for 'Halisbey' and $30.17 \%$ for 'NC-7'), and the greatest linoleic acid contents were obtained from $\mathrm{I}_{100}$ irrigation level (33.60\% for 'Halisbey' and 32.62\% for NC-7) (Table 3). In 2018, 'Halisbey" and NC-7' cultivars also exhibited the same responses. The lowest linoleic acid content was observed in the lowest irrigation level $\left(\mathrm{I}_{50}\right)$. The greatest linoleic acid content was observed in $\mathrm{I}_{75}$ treatment of 'Halisbey' (36.43\%) and $\mathrm{I}_{100}$ treatment of 'NC-7' (29.37\%). In the four-day irrigation interval, linoleic acid contents of these two cultivars had similar variations based on irrigation levels. The lowest linoleic acid content was obtained from $\mathrm{I}_{75}$ treatment (Figure $2 \mathrm{c}$ and $\mathrm{d})$.

In Sultan cultivar, similar regression curves were obtained for linoleic acid contents in both years and irrigation intervals. The lowest linoleic acid contents at a two-day irrigation interval of 2017 and both two and four-day irrigation intervals of 2018 were observed in the $\mathrm{I}_{75}$ irrigation level (Figure $2 \mathrm{c}$ and $\mathrm{d}$ ).

The total saturated fatty acid content of the cultivars was greater in 2018 than in 2017. Among the present cultivars, ' $\mathrm{NC}-7$ ' had the greatest total saturated fatty acid content. Considering the year $\mathrm{x}$ cultivar $\mathrm{x}$ irrigation interval $\mathrm{x}$ irrigation level interactions, the greatest total saturated fatty acid ratio $(82.74 \%)$ was obtained from the $\mathrm{I}_{100}$ irrigation level of the second year at a two-day irrigation interval of NC-7 cultivar (Table 3).

Oleic acid is the second unsaturated fatty acid of peanuts, and oleic acid contents of peanuts were reported as between 20 - 45\% by Liu et al. (2008) and between 20.9 - 43.2\% by Carrin and Carelli (2010). The change in linoleic acid content based on genetics and environmental factors (drought stress) directly reflects on oleic acid content. In other words, decreasing oleic acid contents were observed with increasing linoleic acid contents (Akram et al., 2018). Just because of significant correlations between these fatty acids, some researchers indicated oleic/linoleic acid ratio $(\mathrm{O} / \mathrm{L})$ as a significant quality parameter. It was reported in previous studies that plants under drought stress and plants grown in cool regions generally had low $\mathrm{O} / \mathrm{L}$ ratios (Hashim et al., 1993; Dwivedi et al., 1993).

Palmitic acid was the major saturated fatty acid of peanut cultivars. For palmitic acid contents, 'Halisbey' cultivar exhibited similar reactions against different irrigation levels in two and four-day irrigation intervals of 2017 and 2018 (Figure 2 and f). In a two-day irrigation interval of 'Halisbey' cultivar, the lowest palmitic acid content was obtained from the $\mathrm{I}_{50}$ irrigation level (10.28\% in 2017 and $10.21 \%$ in 2018) (Table 3 and 4 ). The greatest palmitic acid ratios of the same cultivar were obtained from $\mathrm{I}_{75}$ treatment in a two-day irrigation interval and $I_{100}$ treatment in a four-day irrigation interval. For palmitic acid content, the most extreme reactions to entire experimental factors were presented by 'NC-7' and 'Sultan' cultivars. In regression analysis, the lowest coefficient of determination values was identified in two and four-day irrigation intervals of these two cultivars (Figure $2 \mathrm{e}$ and $\mathrm{f}$ ).

In both years, in a two-day irrigation interval, the palmitic acid content of 'NC-7' cultivar initially increased, then decreased with increasing irrigation levels. In the four-day irrigation interval, palmitic acid 
contents increased in both years with increasing irrigation levels. The lowest palmitic acid content in this irrigation interval was obtained from $\mathrm{I}_{50}$ and the greatest from $\mathrm{I}_{125}$ treatments. In 'Sultan' cultivar, in a two-day irrigation interval, palmitic acid contents increased in both years with increasing irrigation levels, however, in four-day irrigation interval, palmitic acid contents decreased with increasing irrigation levels (Figure $2 \mathrm{e}$ and $\mathrm{f}$ ).

The other unsaturated fatty acids of peanut seeds included arachidic, behenic, lignoceric, and myristic acids. The averages for these fatty acids based on experimental factors are provided in Tables 3 , and 4 and regression curves are presented in Figure 2.

For arachidic acid, regression curves were similar to each other (Figures $3 \mathrm{a}$ and $\mathrm{b}$ ). In both years of the experiments, in two-day irrigation interval, arachidic acid contents decreased in 'NC-7' cultivar and increased in 'Halisbey' and 'Sultan' cultivars with increasing irrigation levels. In the four-day irrigation interval, arachidic acid contents increased in 'NC-7' and 'Sultan' cultivars with increasing irrigation levels in both years. In 'Halisbey' cultivar, in the first year, arachidic acid contents decreased with $\mathrm{I}_{75}$ and $\mathrm{I}_{100}$ treatments, but increased with $\mathrm{I}_{125}$ irrigation level; in the second year, arachidic acid contents decreased with increasing irrigation levels.

In the first year, the lowest behenic acid contents of two-day irrigation intervals were obtained from $\mathrm{I}_{75}$ treatments of 'Halisbey' and 'Sultan' cultivars, and the lowest behenic acid contents of four-day irrigation intervals were obtained from $\mathrm{I}_{50}$ and $\mathrm{I}_{125}$ treatments. In 2018, in a two-day irrigation interval, the lowest behenic acid contents were observed in 'Halisbey' and 'Sultan'; in a four-day irrigation interval, the lowest values were obtained from the $\mathrm{I}_{75}$ irrigation level of 'Halisbey' and 'NC-7' cultivars. In both years, the greatest behenic acid contents of two-day irrigation intervals were obtained from $\mathrm{I}_{125}$ treatment of 'Halisbey' cultivar, and the greatest behenic acid contents of four-day irrigation intervals were obtained from $\mathrm{I}_{125}$ treatment of 'NC-7' cultivar (Figure $3 \mathrm{c}$ and $\mathrm{d}$ ).

For lignoceric acid contents, 'Halisbey' and 'Sultan' cultivars had similar reactions in 2017 and 2018 against irrigation intervals and irrigation levels. In both years, the lignoceric acid content of both cultivars increased in two-day irrigation intervals and decreased in four-day irrigation intervals with increasing irrigation levels. In 'NC-7' cultivar, lignoceric acid contents decreased in two-day irrigation intervals and increased in four-day irrigation intervals with increasing irrigation levels in both years (Figure $3 \mathrm{e}$ and $\mathrm{f}$ ).

Myristic acid was the least common unsaturated fatty acid in present experiments. 'NC-7' and 'Sultan' cultivar and similar changes in myristic acid contents with the years and irrigation intervals. In 'NC-7' cultivar, in a two-day irrigation interval of both years, myristic acid contents initially decreased with irrigation levels but reached the maximum in $\mathrm{I}_{125}$ treatment. In four-day irrigation intervals of the same cultivar, a reverse case was evident, there was an increase in $\mathrm{I}_{50}, \mathrm{I}_{75}$, and $\mathrm{I}_{100}$ treatments, but a decrease in $\mathrm{I}_{125}$ treatment (Table 3 and 4). In two-day irrigation intervals of 'Halisbey' cultivar, myristic acid contents increased in $\mathrm{I}_{50}, \mathrm{I}_{75}$, and $\mathrm{I}_{100}$ treatments and decreased in $\mathrm{I}_{125}$ treatment in both years (Figure $3 \mathrm{~g}$ and $\mathrm{h}$ ). In 'Sultan' cultivar, myristic acid contents did not change significantly with irrigation levels in two and four-day irrigation intervals of the first year; significant decreases were observed in myristic acid content, especially in four-day irrigation intervals of the second year with increasing irrigation levels.

The biplot graphs generated for visual assessment of the relationships between the fatty acids of the cultivars based on irrigation intervals and irrigation levels are presented in Figure 4. Each one of the present biplots explained over $60 \%$ of total variations. Since Singkham et al. (2010) used the oleic/linoleic acid ratio as a quality criterion for peanuts, $\mathrm{O} / \mathrm{L}$ ratio is presented in Figure 4.

As can be seen in Figure 4a, investigated fatty acids formed four groups in 'Halisbey' cultivar in the first year. There were significant negative correlations between G-IV (linoleic acid) and G-II (oleic acid and oleic/linoleic acid ratio); between G-III (arachidic acid, TUSFA) and G-I (the other saturated fatty acids, TSFA, and oil ratio) (Figure 4a). In the second year, TUSFA alone formed Group-IV and had negative correlations with Group-II (lignoceric, palmitic acid, oil ratio, and TSFA). Group-III was composed of oleic acid and oleic/linoleic acid ratio and had negative correlations with Group-I (Figure 4b). In both years of 'Halisbey' cultivar, the group including oleic acid (G-II in the first year and G-III in the second year) negatively correlated with the group, including oil ratio (G-I in the first year and G-II in the second year). Such a finding 
indicated that the treatments to increase the oil ratio of this cultivar would reduce the oleic/linoleic acid ratio. Like this, in both years, 2D- $\mathrm{I}_{50}, 4 \mathrm{D}-\mathrm{I}_{50}$, and $4 \mathrm{D}-\mathrm{I}_{75}$ treatments increased oleic acid contents, and 4D-I $\mathrm{I}_{100}$ treatment increased oil content in both years (Table 3 and 4 ).

In NC-7 cultivar, the interactions between oil ratio and fatty acids were similar in both years. In the first year, TUSFA and myristic acid alone formed single groups. In the second year, myristic acid and TUSFA were placed in the same group. In the first year, there were significant negative correlations between Group- $\mathrm{V}$ (oleic acid, oil content, oleic/linoleic acid ratio) with the greatest vector length and Group-II (linoleic acid). There were significant negative correlations between Group-IV (TUSFA) and Group-I (arachidic, palmitic, lignoceric, behenic acids, and TSFA) (Figure 4c). In the second year, there were significant negative correlations between Group-V (oleic and oleic/linoleic acid ratio) and Group-II (linoleic acid) and between Group-III (TUSFA and Myristic) and Group-I (oil content, arachidic, palmitic, lignoceric, behenic acids, and TSFA) (Figure 4d). In 'NC-7' cultivar, the oil ratio had significant positive correlations with oleic acid and oleic/linoleic acid ratios. Thus, they were placed into the same attribute group $(\mathrm{G}-\mathrm{V})$ in the first year. In the second year, the level of correlation of oil ratio with oleic acid and oleic/linoleic ratios was lower. Thus, oil ratio was placed into a different group. In NC-7 cultivar, $4 \mathrm{D}-\mathrm{I}_{50}, 2 \mathrm{D}-\mathrm{I}_{50}$, and $4 \mathrm{D}-\mathrm{I}_{75}$ treatments were prominent for high oil and oleic acid content in the first year, and besides these treatments, $4 \mathrm{D}-\mathrm{I}_{100}$ was also prominent for both oil ratio and oleic acid content in the second year (Figure 4c and d).

In 'Sultan' cultivar, there were significant negative correlations between Group-IV (TUSFA and myristic) and Group-II (behenic, TSFA, and arachidic) and between Group-III (oleic, oil content, oleic/linoleic acid ratio) and Group-I (linoleic, lignoceric, and palmitic) in the first year (Figure 4e). In the second year, arachidic acid alone formed a group (Group-3), and this group had significant negative correlations with Group-V (TUSFA, myristic) and Group-I (linoleic). The greatest negative correlation was observed between Group-I (linoleic) and Group-IV (oil content, oleic acid, and oleic/linoleic acid ratio) (Figure 4f). The most stable and positive correlations between oil content and oleic acid were observed in the 'Sultan' cultivar. In this cultivar, oil content, oleic acid, and oleic/linoleic acid ratio were placed in the same attribute group. The 2D-I 75 treatment had positive correlations with this attribute group and placed in the closest position. Such a case indicated that $2 \mathrm{D}-\mathrm{I}_{75}$ was the best irrigation combination for high oil content and thus high oleic acid content in Sultan cultivar (Figure 4e and f).

High oleic acid content is the primary quality attribute in peanuts, as can be inferred from the present findings that oleic acid contents varied with the cultivars and environmental conditions. When an appropriate irrigation program was applied, high oleic acid contents could be achieved based on the genetic potential of the cultivar. As can be seen in Figure 4, the greatest oleic contents in 'Halisbey' and 'NC-7' cultivars were obtained from $\mathrm{I}_{50}$ treatment of two and four-day irrigation intervals. In 'Sultan' cultivar, the greatest oleic acid content was obtained from $\mathrm{I}_{75}$ treatment of two and four-day irrigation intervals of the second year.

Three peanut cultivars used in the present experiments were the most common cultivars of Turkey. As a general average, the greatest oleic acid content (51.90\%) was obtained from 'NC-7' cultivar, and it was followed by 'Sultan' (47.12\%) and 'Halisbey' (46.40\%) cultivars. Similarly, in peanut samples taken from common peanut-cultivated regions, 'NC-7' cultivar had greater oleic acid content than 'Sultan' and 'Halisbey' cultivars (Kalefetoğlu et al., 2018).

Nutritional quality, taste, and shelf life of peanut products and seeds largely depend on relative quantities of saturated fatty acids (SFA), mono-unsaturated fatty acids (MUFA) and poly-unsaturated fatty acids (PUFA). High oleic acid contents play a significant role both in human health and thermo-oxidative stability of peanuts for industrial purposes (Nawade et al., 2018). Singkham et al. (2010) indicated that genetics was the most significant factor affecting the fatty acid composition of peanuts, genotype $\mathrm{x}$ environment interactions were also significant for oleic, linoleic acids and $\mathrm{O} / \mathrm{L}$ ratio and genetic effects especially on oleic acid contents may decrease based on $(\mathrm{GxE})$ interactions. Climate, irrigation, and soil conditions are effective primary factors in peanut cultivation. During the seed ripening stage, environmental factors generally increase oleic acid contents and decrease the other fatty acid quantities (Sanders, 1980). In the present study, significant 
variations were observed in the oleic contents of three peanut cultivars with irrigation intervals and irrigation levels. In the two-day irrigation interval, oleic acid contents decreased in 'NC-7', and 'Halisbey' cultivars with increasing irrigation levels and the greatest oleic acid content in Sultan cultivar were achieved in the $\mathrm{I}_{75}$ irrigation level. In a four-day irrigation interval, the response of cultivars for oleic acid content varied with the years and irrigation levels. In this irrigation interval, the greatest oleic acid content was obtained fromI ${ }_{75}$ treatment of 'NC-7' cultivar. The oleic acid contents were generally greater in 2018 than in 2017. Such a case was mostly attributed to climate factors. It was reported in a previous study that high temperatures during the seed development phase reduced linoleic acid content and increased oleic acid content of peanut oil (Casini et al., 2003). Plant tolerance of high temperatures largely depends on soil moisture, thus irrigation treatments. The oleic and stearic acid content of peanuts grown in rainy seasons was greater than the peanuts grown in dry seasons and linoleic, and palmitic acid contents also increased in rainy seasons (Singkham et al., 2010). Chaiyadee et al. (2013) reported that droughts improved the oil quality of peanuts, increased oleic acid contents, genotype $\mathrm{x}$ environment interactions influenced fatty acid composition, resistant and sensitive genotypes exhibited similar response to droughts and recommended further research about the effects of droughts on oleic acid content.

Since irrigations have quite significant effects on peanut oil quality, effects of irrigation intervals and irrigation levels on oil content and fatty acid composition of three commonly cultivated peanut cultivars of Turkey were put forth in this study. Especially the effects of irrigation interval $\mathrm{x}$ irrigation level interactions on fatty acid composition were investigated. In previous studies conducted on peanuts, either irrigation intervals or irrigation levels, were investigated alone. Together with different peanut cultivars, two factors were not considered together in previous studies. Previous studies mostly focused on a single cultivar with different irrigation intervals or with different irrigation levels. Aydinsakir et al. (2019) applied different water-deficit levels to 'NC-7' peanut cultivar under Mediterranean conditions and reported that oleic acid contents decreased with increasing irrigation levels, linoleic acid contents increased until $\mathrm{I}_{75}$ treatment and decreased in $I_{100}$ and $I_{125}$ treatments. In another study, decreasing oleic contents were reported with increasing irrigation levels, and the greatest linoleic acid contents were reported for $\mathrm{I}_{75}$ and $\mathrm{I}_{100}$ treatments (Boydak et al., 2010).

\section{Conclusions}

Regression graphs facilitated visual assessment of oil content, and fatty acid composition of peanut cultivars and biplot graphs facilitated the assessment of cultivar $\mathrm{x}$ years $\mathrm{x}$ irrigation interval $\mathrm{x}$ irrigation level interactions. With the aid of biplot graphs, the best irrigation interval $\mathrm{x}$ irrigation level combination for each attribute group was tried to be identified. In two and four-day irrigation intervals, the greatest oil contents were generally achieved in $\mathrm{I}_{75}$ and $\mathrm{I}_{100}$ irrigation levels. Recommendations of cultivar, irrigation interval, and irrigation level were provided for high oleic acid contents. It was concluded based on present findings that high oleic acid contents could be achieved based on genetic potential of the cultivars with a proper irrigation program; $\mathrm{I}_{50}$ treatment could be used at two or four-day irrigation intervals in 'Halisbey', and 'NC-7' cultivars and $\mathrm{I}_{75}$ treatment could be used in four-day irrigation interval in 'Sultan' cultivar to get high oleic acid contents under sandy soil conditions.

\section{Authors' Contributions}

Conceptualization: SA and IT; Methodology: IT; Validation: SA, MK and IT; Formal analysis: SA and MK; Investigation: SA, IT and MK; Data curation: MK, KK and ASB; Funding acquisition: IT; Writing: SA; Review and editing; SA, MK and IT. All authors read and approved the final manuscript. 


\section{Acknowledgements}

We would like to thank the staff of Balıkesir Metropolitan Municipality Rural Development Department for their assistance in conducting the field trials of this research.

\section{Conflict of Interests}

The authors declare that there are no conflicts of interest related to this article.

\section{References}

Abbas Z, Kumar A, Kumar A (2018). Peanut agriculture and production technology: integrated nutrient management. Apple Academic Press, USA, pp 114-122.

Akram NA, Shafiq F, Ashraf M (2018). Peanut (Arachis hypogaea L.): A prospective legume crop to offer multiple health benefits under changing climate. Comprehensive Reviews in Food Science and Food Safety 17:1325-1338. https://doi.org/10.1111/1541-4337.12383

Akhtar S, Khalid N, Ahmed I, Shahzad A, Suleria HAR (2014). Physicochemical characteristics, functional properties, and nutritional benefits of peanut oil: a review. Critical Reviews in Food Science and Nutrition 54:1562-1575. https://doi.org/10.1080/10408398.2011.644353

Amir Y, Benbelkacem T, Hadni L, Youyou A (2005). Effect of irrigation and fertilization on characteristics of peanut seeds cultivated near Tizi-Ouzou. Electronic Journal of Environmental, Agricultural and Food Chemistry 4:879-885.

Andersen PC, Gorbet DW (2002). Influence of year and planting date on fatty acid chemistry of high oleic acid and normal peanut genotypes. Journal of Agricultural and Food Chemistry 50:1298-1305. https://doi.org/10.1021/jf0113171

Arıoglu H, Bakal H, Güllüoğlu L, Kurt C, Onat B (2016). The determination of some important agronomical and quality properties of peanut varieties in main crop conditions. Journal of Central Research Institute 25:24-29.

Arioglu H (2014). The oil seed crops growing and breeding. The publication of University of Cukurova, Faculty of Agriculture, Adana, Turkey pp 204.

Aydinsakir K, Dinc N, Buyuktas D, Bastug R, Toker R (2016). Assessment of different irrigation levels on peanut crop yield and quality components under Mediterranean conditions. Journal of Irrigation and Drainage Engineering 142:34-39. https://doi.org/10.1061/(ASCE)IR.1943-4774.0001062

Boydak E, Karaaslan D, Türkoğlu H (2010). The effect of different nitrogen and irrigation levels on fatty acid composition of peanut oils. Turkish Journal of Field Crops 15:29-33.

Carrin ME, Carelli AA (2010). Peanut oil: Compositional data. European Journal of Lipid Science and Technology 112:697-707. https://doi.org/10.1002/ejlt.200900176

Casini C, Dardanelli JL, Mart'ınez MJ, Balzarini M, Borgogno CS, Nassetta M (2003). Oil quality and sugar content of peanuts (Arachis hypogaea) grown in Argentina: Their relationship with climatic variables and seed yield. Journal of Agricultural and Food Chemistry 51:6309-6313. https://doi.org/10.1021/jf030183j

Chaiyadee S, Jogloy SP, Songsri P, Singkham N, Vorasoot N, Sawatsitang P, Patanothai A (2013). Soil moisture affects fatty acids and oil quality parameters in peanut. International Journal of Plant Production 7:81-96.

Chilliard Y, Rouel J, Guillouet P (2013). Goat alpha-s1 casein genotype interacts with the effect of extruded linseed feeding on milk fat yield, fatty acid composition and post-milking lipolysis. Animal Feed Science and Technology 185. https://doi.org/140-149. 10.1016/j.anifeedsci.2013.08.002

Christie WW (1990). Gas chromatography and lipids. The Oil Press, Glasgow, pp 302.

Davis JP, Price KM, Dean LL, Sweigart DS, Cottonaro JM, Sanders TH (2016). Peanut oil stability and physical properties across a range of industrially relevant oleic acid/ linoleic acid ratios. Peanut Science 43:1-11.

Dwivedi SL, Nigam SN, Jambunathan R, Sahrawat KL, Nagabhushanam GVS, Raghunath K (1993). Effect of genotypes and environments on oil content and oil quality parameters and their correlation in peanut (Arachis hypogaea L.). Peanut Science 20:84-89. 
Dwivedi SL, Nigam SN, Nageswara Rao RC, Singh U, Rao KVS (1996). Effect of drought on oil, fatty acids and protein contents of groundnut (Arachis hypogaea L.) seeds. Field Crops Research 48:125-133. https://doi.org/10.1016/S0378-4290(96)01027-1

Florkowski WJ (2012). Groundnut production and trade. In: Smartt J (Ed). The Groundnut Crop: A Scientific Basis for Improvement. Springer Science \& Business Media, pp 1-23.

Hashim IB, Koehler PE, Eitenmiller RR, Kvien CK (1993). Fatty acid composition and tocopherol content of drought stressed florunner peanuts. Peanut Science 20:21-24.

Hinds MJ (1995). Fatty acid composition of Caribbean-grown peanuts at 3 maturity stages. Food Chemistry 53:7-14. https://doi.org/10.1016/0308-8146(95)95779-6

Isleib TG, Tilman BL, Patte HE, Sanders TH, Hendrix KW, Dean LO (2008). Genotype-by-environment interaction for seed composition traits of breeding lines in the uniform peanut performance test. Peanut Science 35:130138.

Janila P, Pandey MK, Shasidhar Y, Variath MT, Sriswathi M, Khera P (2016). Molecular breeding for introgression of fatty acid desaturase mutant alleles (ahFAD2A and ahFAD2B) enhances oil quality in high and low oil containing peanut genotypes. Plant Science 242:203-213. https://doi.org/10.1016/j.plantsci.2015.08.013

Kaplan M, Karaman K, Kardes YM, Kale H (2019). Phytic acid content and starch properties of maize (Zea mays L.): Effects of irrigation process and nitrogen fertilizer. Food Chemistry 283:375-380. https://doi.org/10.1016/j.foodchem.2019.01.029

Kalefetoğlu T, Macar O, Çil AN, Oluk CA, Çil A (2018). Assessment of peanut (Arachis hypogaea L.) Genotypes in terms of some nutritional and antioxidant parameters. Süleyman Demirel Üniversitesi Fen Bilimleri Enstitüsü Dergisi 22:1175-1181.

Kokten K, Kaplan M, Seydosoğlu S, Ozdemir S, Boydak E (2014). Determination of animal nutrition values of dry biomass of different peanut (Arachis hypogaea) varieties. Turkish Journal of Agricultural and Natural Science 1:156-160.

Langat MC, Okiror MA, Ouma JP, Gesimba RM (2006). The effect of intercropping groundnut (Arachis hypogea L.) with sorghum (Sorghum bicolor L. Moench) on yield and cash income. Agricultura Tropica Subtropica 39:8790.

Liu D, Sun W, Yu B, Liu R, Fang X, Zhang A (2008). Simultaneous preparation of peanut oil and defatted protein powder by low-temperature prepressing, extraction and low-temperature desolventizing. China Oils and Fats 33:13-15.

Nawade B, Mishra GP, Radhakrishnan T, Dodia SM, Ahmad S, Kumar A, Kundu R (2018). High oleic peanut breeding: Achievements, perspectives, and prospects. Trends in Food Science \& Technology 78:107-119. https://doi.org/10.1016/j.tifs.2018.05.022

O’Brien RD (2004). Fats and oils. Formulating and processing for applications. CRC Press, Boca Raton, USA.

Ozcan MM (2010). Some nutritional characteristics of kernel and oil of peanut (Arachis hypogaea L.). Journal of Oleo Science 59. https://doi.org/1-5. 10.5650/jos.59.1

Patel GN, Patel PT, Patel PH (2008). Yield, water use efficiency and moisture extraction pattern of summer groundnut as influenced by irrigation schedules, sulfur levels and sources. Journal of SAT Agricultural Research 6:1-4.

Reddy TY, Reddy VR, Anbumozhi V (2003). Physiological responses of groundnut (Arachis hypogaea L.) to drought stress and its amelioration: A critical review. Plant Growth Regulation 41:75-88. https://doi.org/10.1023/A:1027353430164

Sanders TH (1980). Fatty acid composition of lipid classes in oils from peanuts differing in variety and maturity. Journal of the American Oil Chemists Society 57:12-15. https://doi.org/10.1007/BF02675517

SAS Institute (2014). SAS User's Guide, SAS Inst., Cary.

Sezen SM, Yucel S, Tekin S, Yildiz M (2019). Determination of optimum irrigation and effect of deficit irrigation strategies on yield and disease rate of peanut irrigated with drip system in Eastern Mediterranean. Agricultural Water Management 221:211-219. https://doi.org/10.1016/j.agwat.2019.04.033

Shinde BM, Laware SL (2010). Effect of drought stress on agronomic and yield contributing characters in groundnut (Arachis hypogaea L.). Asian Journal of Experimental Biological Sciences 1:968-971.

Singkham N, Jogloy S, Kesmala T, Swatsitang P, Jaisil P, Puppala N (2010). Genotypic variability and genotype by environment interactions in oil and fatty acids in high, intermediate, and low oleic acid peanut genotypes. Journal of Agricultural and Food Chemistry 58(10):6257-6263. https://doi.org/10.1021/jf903728e

Tas I (2017). Su Kirliliğinin Tarıma Etkileri. Turk Tarım 63:39-43. 
Toomer OT (2018). Nutritional chemistry of the peanut (Arachis hypogaea). Critical Reviews in Food Science and Nutrition 58(17):3042-3053. https://doi.org/10.1080/10408398.2017.1339015

Yan W (2001). GGEBiplot-a Windows application for graphical analysis of multi-environment trial data and other types of two-way data. Agronomy Journal 93(11):1111-1118. https://doi.org/10.2134/agronj2001.9351111X

Yao C, Lei T, Yang P, Gao N, Mao J (2003). Experimental study on dynamic root and canopy development of peanuts under different water regimes. ASAE Annual International Meeting, ASAE, St. Joseph, MI.

Yol E, Uzun B (2018). Influences of genotype and location interactions on oil, fatty acids and agronomical properties of groundnuts. Grasas y Aceites 69:276-286. https://doi.org/10.3989/gya.0109181

Yol E, Ustun R, Golukcu M, Uzun B (2017). Oil content, oil yield and fatty acid profile of groundnut germplasm in Mediterranean climates. Journal of the American Oil Chemists' Society 94:787-804. https://doi.org/10.1007/s11746-017-2981-3

Young CT, Worthington RE, Hammons RO, Matlock RS, Waller GR, Morrison RD (1974). Fatty acid composition of Spanish peanut oils as influenced by planting location, soil moisture conditions, variety, and season. Journal of the American Oil Chemists' Society 51:312-315.

Young T (1996). Peanut oil. In: Hui YH (Ed). Bailey’s Industrial Oil and Fat Products. John \& Wiley Sons, New York, USA, pp 377-392.

Zhao X, Chen J, Du F (2012). Potential use of peanut by-products in food processing: A review. Journal of Food Science and Technology 49(5):521-529. https://doi.org/10.1007/s13197-011-0449-2

Zhu H, Lamb MC, Butts CL, Blankenship PD (2004). Improving peanut yield and grade with surface drip irrigation in undulating fields. Transactions of the ASAE 47:99-106.

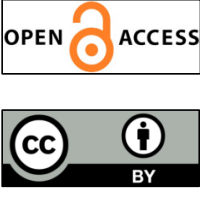

The journal offers free, immediate, and unrestricted access to peer-reviewed research and scholarly work. Users are allowed to read, download, copy, distribute, print, search, or link to the full texts of the articles, or use them for any other lawful purpose, without asking prior permission from the publisher or the author.

License - Articles published in Notulae Botanicae Horti Agrobotanici Cluj-Napoca are Open-Access, distributed under the terms and conditions of the Creative Commons Attribution (CC BY 4.0) License. (c) Articles by the authors; UASVM, Cluj-Napoca, Romania. The journal allows the author(s) to hold the copyright/to retain publishing rights without restriction. 